Pure and Applied Mathematics Quarterly

Volume 9, Number 4

(Special Issue: In memory of

Andrey Todorov, Part 1 of 3 )

$643-664,2013$

\title{
Pseudo-Effective Classes and Pushforwards
}

\author{
Olivier Debarre, Zhi Jiang and Claire Voisin \\ In memory of Andrey Todorov
}

\begin{abstract}
Given a morphism between complex projective varieties, we make several conjectures on the relations between the set of pseudo-effective (co)homology classes which are annihilated by pushforward and the set of classes of varieties contracted by the morphism. We prove these conjectures for classes of curves or divisors. We also prove that one of these conjectures implies Grothendieck's generalized Hodge conjecture for varieties with Hodge coniveau at least 1.
\end{abstract}

Keywords: Pseudo-effective classes, Hodge conjecture.

\section{INTRODUCTION AND CONJECTURES}

Let $X$ and $Y$ be complex projective varieties and let $\phi: X \rightarrow Y$ be a morphism. We say that an irreducible subvariety $Z$ of $X$ is contracted by $\phi$ if $\operatorname{dim}(\phi(Z))<\operatorname{dim}(Z)$. If $k:=\operatorname{dim}(Z)$, this is equivalent to saying that the class $[Z] \in H_{2 k}(X, \mathbf{R})$ is in the kernel of the push-forward morphism

$$
\phi_{*}: H_{2 k}(X, \mathbf{R}) \rightarrow H_{2 k}(Y, \mathbf{R}) .
$$

In this article, we discuss some extensions of this equivalence to more general cycle classes. In $H_{2 k}(X, \mathbf{R})$, classes of irreducible subvarieties of $X$ generate a closed convex cone, called the pseudo-effective cone, whose elements are called

Received January 16, 2013.

2010 Mathematics Subject Classification. 14C25, 14C30. 
pseudo-effective classes. We are interested in pseudo-effective classes annihilated by $\phi_{*}$ and make the following conjecture.

Conjecture A. Let $X$ and $Y$ be complex projective varieties and let $\phi: X \rightarrow Y$ be a morphism. Then any pseudo-effective class $\alpha \in H_{2 k}(X, \mathbf{R})$ such that $\phi_{*} \alpha=$ 0 in $H_{2 k}(Y, \mathbf{R})$ belongs to the real vector space (resp. the closed convex cone) spanned by classes of $k$-dimensional subvarieties of $X$ which are contracted by $\phi$.

The "resp." form of the conjecture is obviously stronger and will be refered to as "Strong Conjecture A," as opposed to "Weak Conjecture A."

Now assume moreover that $X$ and $Y$ are smooth and set $c:=\operatorname{dim}(X)-\operatorname{dim}(Y)$. Using Poincaré duality, one defines Gysin morphisms

$$
\phi_{*}: H^{2 l}(X, \mathbf{R}) \rightarrow H^{2 l-2 c}(Y, \mathbf{R})
$$

as the compositions

$$
H^{2 l}(X, \mathbf{R}) \stackrel{P D_{X}}{\longrightarrow} H_{2 \operatorname{dim}(X)-2 l}(X, \mathbf{R}) \stackrel{\phi_{*}}{\longrightarrow} H_{2 \operatorname{dim}(X)-2 l}(Y, \mathbf{R}) \stackrel{P D_{Y}}{\longrightarrow} H^{2 l-2 c}(Y, \mathbf{R}) .
$$

Any subvariety $Z$ of $X$ has a cohomology class, and $Z$ is contracted by $\phi$ if and only if this class is annihilated by the suitable Gysin morphism. Conjecture A then takes the following form.

Conjecture B. Let $X$ and $Y$ be smooth complex projective varieties and let $\phi: X \rightarrow Y$ be a morphism. Then any pseudo-effective class $\alpha \in H^{2 l}(X, \mathbf{R})$ such that $\phi_{*} \alpha=0$ belongs to the real vector space (resp. the closed convex cone) spanned by classes of $l$-codimensional subvarieties of $X$ which are contracted by $\phi$.

Finally, a variant of Conjecture B is obtained by modifying the positivity notion used: instead of algebraic pseudo-effectivity, we can consider weak, that is, analytic pseudo-effectivity, where we say that an algebraic cycle is weakly pseudo-effective if its cohomology class is the class of a positive current. Pseudoeffective cycle classes are weakly pseudo-effective, but the converse is unknown except in the divisor case (see [De1]) and the curve case, which follows from Kleiman's theorem. More generally, we will say that a real cohomology class is weakly pseudo-effective if it is a limit of weakly pseudo-effective cycle classes.

We can consider the following strengthening of the weak form of Conjecture B. 
Conjecture C. Let $X$ and $Y$ be smooth complex projective varieties and let $\phi: X \rightarrow Y$ be a morphism. Then any weakly pseudo-effective class $\alpha \in H^{2 l}(X, \mathbf{R})$ such that $\phi_{*} \alpha=0$ belongs to the real vector space spanned by classes of $l$ codimensional subvarieties of $X$ which are contracted by $\phi$.

Evidence for these conjectures is provided in Section 2, where we prove them when no subvarieties are contracted by $\phi$ (i.e., when $\phi$ is finite). In Section 3, we prove that we may assume that $\phi$ is surjective and $Y$ is a projective space. We show furthermore that Conjecture A can be reduced to the case where $\phi$ has connected fibers (a reduction that we were unable to perform in the smooth setting).

Our first main result is then the following.

Theorem 1.1. For curves $(k=1$ and $l=\operatorname{dim}(X)-1)$, Weak Conjecture $A$, Strong Conjecture B, and Conjecture $C$ all hold true.

For divisors $(l=1)$, Strong Conjecture $B$ holds true.

In other words, if $\phi: X \rightarrow Y$ is a morphism between projective varieties, a pseudo-effective degree-2 homology class annihilated by $\phi_{*}$ belongs to the vector space generated by classes of contracted curves. If $X$ is smooth, it belongs to the closed convex cone generated by these classes and the same result holds for divisor classes.

The proof of this theorem occupies Sections 4 (for the curve case) and 5 (for the divisor case).

Finally, we show in Section 6 that Conjecture $C$ implies a strong form of the generalized Hodge conjecture for smooth projective varieties $X$ such that $H^{i, 0}(X)=0$ for $i>0$ (see Theorem 6.4). This is similar to the implication proved in [V2], where effectivity of certain strongly positive cycles on Fano varieties of lines of complete intersections is proved to imply the generalized Hodge conjecture for coniveau- 2 complete intersections in projective spaces.

Acknowledgements. We thank Mihai Fulger for pointing out an error in a previous version of this article. 


\section{The Finite CASE}

A first evidence for our conjectures is provided by the case where no subvarieties are contracted by $\phi$.

Proposition 2.1. Let $\phi: X \rightarrow Y$ be a finite morphism between projective varieties. If $\alpha \in H_{2 k}(X, \mathbf{R})$ is pseudo-effective and $\phi_{*} \alpha=0$ in $H_{2 k}(Y, \mathbf{R})$, then $\alpha=0$.

Proof. Let $L$ be an ample line bundle on $Y$. Then $\phi^{*} L$ is ample on $X$. Choose a projective embedding $i: X \hookrightarrow \mathbf{P}^{N}$ such that $i^{*} \mathscr{O}_{\mathbf{P}^{N}}(1)=\phi^{*} L^{\otimes m}$ for some $m>0$, and let $\omega \in A^{1,1}\left(\mathbf{P}^{N}, \mathbf{R}\right)$ be a positive Chern form of $\mathscr{O}_{\mathbf{P}^{N}}(1)$. As the class [ $\left.\omega\right]$ of $\omega$ is $c_{1}\left(\mathscr{O}_{\mathbf{P}^{N}}(1)\right)$, its restriction to $X$ is $c_{1}\left(\phi^{*}\left(L^{\otimes m}\right)\right)$, hence we have

$$
\left\langle\alpha,\left.[\omega]^{k}\right|_{X}\right\rangle_{X}=\left\langle\phi_{*} \alpha, c_{1}\left(L^{\otimes m}\right)^{k}\right\rangle_{Y}=0 .
$$

On the other hand, we have

$$
\left\langle\alpha,\left.[\omega]^{k}\right|_{X}\right\rangle_{X}=\left\langle i_{*} \alpha,[\omega]^{k}\right\rangle_{\mathbf{P}^{N}} .
$$

Note that $\alpha$ being pseudo-effective, $i_{*} \alpha$ is pseudo-effective, and thus $i_{*} \alpha$ is the class of a positive current $T$ of type $(k, k)$. We now use the following standard result.

Lemma 2.2. Let $T$ be a compactly supported positive current of type $(k, k)$ on a complex manifold endowed with a positive $(1,1)$-form $\omega$. If $\int_{T} \omega^{k}=0$, then $T=0$.

We apply this lemma to a neighborhood $U$ of $X$ in $\mathbf{P}^{N}$ which is a deformation retract of $X$. Let us denote by $i_{U}$ the inclusion of $X$ in $U$. The homology class $i_{U *} \alpha \in H_{2 k}(U, \mathbf{R})$ is the homology class of the compactly supported closed positive current $T$ on $U$, hence it is 0 by the lemma above. On the other hand, $i_{U *}: H_{\bullet}(X, \mathbf{R}) \stackrel{\sim}{\rightarrow} H_{\bullet}(U, \mathbf{R})$ is an isomorphism, because $U$ retracts onto $X$. Thus $\alpha=0$ in $H_{2 k}(X, \mathbf{R})$.

Remark 2.3. In this proof, we used an embedding of $X$ into $\mathbf{P}^{N}$ because $X$ could be singular. When $X$ is smooth, we can of course directly use Lemma 2.2 on $X$.

Remark 2.4. The same proof applies in the setting of Conjecture C, which therefore holds true for finite morphisms. 
We use Proposition 2.1 to show the following.

Corollary 2.5. In order to prove Conjecture A, it suffices to prove it for morphisms with connected fibers.

Proof. Let $\phi: X \rightarrow Y$ be a morphism, where $X$ and $Y$ are projective, and let $\alpha \in H_{2 k}(X, \mathbf{R})$ be a pseudo-effective class such that $\phi_{*} \alpha=0$. We use the Stein factorization $\phi=h \circ \psi$, where $\psi: X \rightarrow Z$ has connected fibers and $h: Z \rightarrow Y$ is finite. The class $\psi_{*} \alpha$ is pseudo-effective on $Z$ and it is annihilated by $h_{*}$. By Proposition 2.1, $\psi_{*} \alpha=0$. If Conjecture $\mathrm{A}$ is true for morphisms with connected fibers, $\alpha$ is in the real vector space (resp. in the closed convex cone) spanned by classes of subvarieties of $X$ contracted by $\psi$, hence by $\phi$.

\section{Reduction to the SURJECtive CASE}

Proposition 3.1. In order to prove any of the conjectures stated in the introduction, it suffices to do so when $\phi$ is surjective and $Y$ is a projective space.

Proof. Let us do it for Conjecture B. For the other two conjectures, the proof is similar. Set $Y^{\prime}:=\phi(X) \subset Y$ and $d:=\operatorname{dim}\left(Y^{\prime}\right)$. Let us denote by $\phi^{\prime}: X \rightarrow Y^{\prime}$ the surjective morphism induced by $\phi$. We choose a rational map $Y \rightarrow \mathbf{P}^{d}$ which restricts to a finite morphism $\pi: Y^{\prime} \rightarrow \mathbf{P}^{d}$. Such a map is obtained by restricting to $Y \subset \mathbf{P}^{N}$ a general linear projection $\mathbf{P}^{N} \rightarrow \mathbf{P}^{d}$. The morphism $\pi \circ \phi$ is surjective and contracts the same subvarieties as $\phi$. Set $n:=\operatorname{dim}(X)$ and $c:=\operatorname{dim}(X)-\operatorname{dim}(Y)$. If $\phi_{*} \alpha=0$ in $H^{2 l-2 c}(Y, \mathbf{R})$, then for any line bundle $L$ on $Y$, we have

$$
\left\langle\phi_{*} \alpha, c_{1}(L)^{n-l}\right\rangle_{Y}=0
$$

which implies

$$
\left\langle\phi_{*}^{\prime} \alpha, c_{1}\left(\left.L\right|_{Y^{\prime}}\right)^{n-l}\right\rangle_{Y^{\prime}}=0 .
$$

The brackets in the above equations are computed as follows: the class $\phi_{*} \alpha \in$ $H^{2 l-2 c}(Y, \mathbf{R})$ is, by definition of the Gysin morphism $\phi_{*}$, the Poincaré dual on $Y$ of the homology class $\phi_{*}\left(P D_{X}(\alpha)\right) \in H_{2 n-2 l}(Y, \mathbf{R})$, where $P D_{X}(\alpha) \in H_{2 n-2 l}(X, \mathbf{R})$ is the Poincaré dual on $X$ of the cohomology class $\alpha$. The intersection number $\left\langle\phi_{*} \alpha, c_{1}(L)^{n-l}\right\rangle_{Y}$ is then given by the pairing between $H_{2 n-2 l}(Y, \mathbf{R})$ and $H^{2 n-2 l}(Y, \mathbf{R})$. Similarly, the intersection number $\left\langle\phi_{*}^{\prime} \alpha, c_{1}\left(\left.L\right|_{Y^{\prime}}\right)^{n-l}\right\rangle_{Y^{\prime}}$ is given by the pairing between $H_{2 n-2 l}\left(Y^{\prime}, \mathbf{R}\right)$ and $H^{2 n-2 l}\left(Y^{\prime}, \mathbf{R}\right)$. 
We apply (1) to the line bundle $L$ giving the rational projection $Y \rightarrow-\mathbf{P}^{d}$. On $Y^{\prime}$, we have $\left.L\right|_{Y^{\prime}}=\pi^{*} \mathscr{O}_{\mathbf{P}^{d}}(1)$, and thus we get from the projection formula

$$
0=\left\langle\phi_{*}^{\prime} \alpha, c_{1}\left(\left.L\right|_{Y^{\prime}}\right)^{n-l}\right\rangle_{Y^{\prime}}=\left\langle\left(\pi \circ \phi^{\prime}\right)_{*} \alpha, c_{1}\left(\mathscr{O}_{\mathbf{P}^{d}}(1)\right)^{n-l}\right\rangle_{\mathbf{P}^{d}}
$$

where the second intersection number is computed using the pairing between $H_{2 n-2 l}\left(\mathbf{P}^{d}, \mathbf{R}\right)$ and $H^{2 n-2 l}\left(\mathbf{P}^{d}, \mathbf{R}\right)$. Formula $(2)$ says that $\left(\pi \circ \phi^{\prime}\right)_{*}\left(P D_{X}(\alpha)\right)=0$ in $H_{2 n-2 l}\left(\mathbf{P}^{d}, \mathbf{R}\right)$, hence $\left(\pi \circ \phi^{\prime}\right)_{*} \alpha=0$ in $H^{2 d-(2 n-2 l)}\left(\mathbf{P}^{d}, \mathbf{R}\right)$. The morphism $\pi \circ \phi^{\prime}$ is surjective and $X$ and $\mathbf{P}^{d}$ are both smooth.

Hence, if we know Conjecture B when the morphism is surjective onto a projective space, we conclude that the class $\alpha$ belongs to the real vector space (resp. the closed convex cone) spanned by classes of subvarieties contracted by $\pi \circ \phi^{\prime}$, which is the same as the real vector space (resp. the closed convex cone) spanned by classes of subvarieties contracted by $\phi$.

\section{THE CURVE CASE}

The following theorem solves Strong Conjecture B in the curve case. It is a straightforward application of the relative Kleiman criterion for ampleness.

Theorem 4.1. Let $X$ and $Y$ be smooth complex projective varieties and let $\phi$ : $X \rightarrow Y$ be a morphism. Set $n:=\operatorname{dim}(X)$. Then any pseudo-effective class $\alpha \in H^{2 n-2}(X, \mathbf{R})$ such that $\phi_{*} \alpha=0$ belongs to the closed convex cone spanned by classes of curves on $X$ which are contracted by $\phi$.

Before beginning the proof, we introduce some notation. For a smooth projective variety $X$, we denote by $N^{1}(X)$ its Néron-Severi group, i.e., the free abelian group of divisors on $X$ modulo numerical equivalence. By the Lefschetz $(1,1)$-theorem, the real vector space $N^{1}(X)_{\mathbf{R}}:=N^{1}(X) \otimes \mathbf{R}$ is isomorphic to $H^{1,1}(X)_{\mathbf{R}}$.

Similarly, we denote by $N_{1}(X)$ the group of 1-cycles on $X$ modulo numerical equivalence. By the Lefschetz $(1,1)$-theorem and the hard Lefschetz theorem, the real vector space $N_{1}(X)_{\mathbf{R}}:=N_{1}(X) \otimes \mathbf{R}$ is isomorphic to $H^{n-1, n-1}(X)_{\mathbf{R}}$ and it is dual to $N^{1}(X)_{\mathbf{R}}$. It contains the closed convex cone spanned by classes of curves in $X$, called the Mori cone and denoted by $\overline{N E}(X)$.

Proof of the theorem. In the vector space $N_{1}(X)_{\mathbf{R}}$, we have two closed convex cones: the intersection $\mathscr{C}_{1}=\overline{N E}(X) \cap \operatorname{Ker}\left(\phi_{*}\right)$ and the closed convex cone $\mathscr{C}_{2}$ 
spanned by classes of curves contracted by $\phi$. We have $\mathscr{C}_{2} \subset \mathscr{C}_{1}$ and we need to prove that these cones are equal.

We argue by contradiction. If $\mathscr{C}_{2} \subsetneq \mathscr{C}_{1}$, there exists a class $d \in N^{1}(X)_{\mathbf{R}}=$ $N_{1}(X)_{\mathbf{R}}^{\vee}$ such that $d$ is positive on $\mathscr{C}_{2} \backslash\{0\}$, but $d \cdot \alpha<0$ for some $\alpha \in \mathscr{C}_{1}$. We may even choose for $d$ the class of a divisor $D$ on $X$. The divisor $D$ is then $\phi$-ample by the relative Kleiman criterion ([KM, Theorem 1.44]). If $H$ is an ample divisor on $Y$, the divisor $m p^{*} H+D$ is therefore ample for $m \gg 0$. But we have

$$
\left(m p^{*} H+D\right) \cdot \alpha=m\left(H \cdot \phi_{*} \alpha\right)+(d \cdot \alpha)<0,
$$

which, by the usual Kleiman criterion, contradicts $\alpha \in \overline{N E}(X)$.

We now prove Conjecture A in its weak form for degree-2 homology classes. Note that the proof above does not apply because we do not have in the singular case a duality between the real vector space spanned by homology classes of curves and the space $N^{1}(X)_{\mathbf{R}}$. However, the proof given below does apply in the setting of Conjecture $\mathrm{C}$, which therefore holds true in the curve case.

Theorem 4.2. Let $X$ and $Y$ be complex projective varieties and let $\phi: X \rightarrow Y$ be a morphism. Then any pseudo-effective class $\alpha \in H_{2}(X, \mathbf{R})$ such that $\phi_{*} \alpha=0$ in $\mathrm{H}_{2}(Y, \mathbf{R})$ belongs to the real vector space spanned by classes of curves in $X$ which are contracted by $\phi$.

Proof. By Proposition 3.1, we can assume that $Y$ is smooth. Next, we prove the following result.

Lemma 4.3. In order to prove Theorem 4.2, it suffices to show that, under the same assumptions, the class $\alpha$ belongs to the real vector subspace $V$ of $H_{2}(X, \mathbf{R})$ spanned by the $j_{y *}\left(H_{2}\left(X_{y}, \mathbf{R}\right)\right)$, for $y \in Y$, where $j_{y}: X_{y} \hookrightarrow X$ is the inclusion of the fiber $X_{y}$.

Proof. This follows from mixed Hodge theory and is proved as follows. The set of cycle classes in $H_{2}(X, \mathbf{Q})$ is contained in the pure part $W_{\text {pure }} H_{2}(X, \mathbf{Q})$ of the mixed Hodge structure on $H_{2}(X, \mathbf{Q})$, since cycle classes lift to cycle classes on a desingularization of $X$. Choose finitely many points $y_{1}, \ldots, y_{N} \in Y$ such that $V$ is spanned by the spaces $j_{y_{i} *}\left(H_{2}\left(X_{y_{i}}, \mathbf{Q}\right)\right)$, for $1 \leq i \leq N$. The morphism

$$
J:=\sum_{i=1}^{N} j_{y_{i} *}: \bigoplus_{i=1}^{N} H_{2}\left(X_{y_{i}}, \mathbf{Q}\right) \rightarrow H_{2}(X, \mathbf{Q})
$$


is a morphism of mixed Hodge structures, hence is strict for the weight filtration by [D2]. Since $\alpha$ is in $(\operatorname{Im}(J) \otimes \mathbf{R}) \cap W_{\text {pure }} H_{2}(X, \mathbf{R})$, it belongs to $J\left(\bigoplus_{i} W_{\text {pure }} H_{2}\left(X_{y_{i}}, \mathbf{Q}\right)\right) \otimes \mathbf{R}$. Furthermore, the morphism

$$
J: \bigoplus_{i} W_{\text {pure }} H_{2}\left(X_{y_{i}}, \mathbf{Q}\right) \rightarrow W_{\text {pure }} H_{2}(X, \mathbf{Q})
$$

is a morphism of polarized Hodge structures, hence maps the space of Hodge classes $\operatorname{Hdg}_{2}\left(\bigoplus_{i} W_{\text {pure }} H_{2}\left(X_{y_{i}}, \mathbf{Q}\right)\right)$ onto the set of Hodge classes belonging to $\operatorname{Hdg}_{2}\left(W_{\text {pure }} H_{2}(X, \mathbf{Q})\right)$ and to $\operatorname{Im}(J)$. If our class $\alpha$ belongs to

$$
\left(\operatorname{Hdg}_{2}\left(W_{\text {pure }} H_{2}(X, \mathbf{Q})\right) \cap \operatorname{Im}(J)\right) \otimes \mathbf{R},
$$

it thus belongs to the space

$$
\left(\bigoplus_{i=1}^{N} j_{y_{i} *}\left(\operatorname{Hdg}_{2}\left(W_{\text {pure }} H_{2}\left(X_{y_{i}}, \mathbf{Q}\right)\right)\right)\right) \otimes \mathbf{R} \subset H_{2}(X, \mathbf{R}) .
$$

To conclude, it suffices to recall that Hodge classes in $\operatorname{Hdg}_{2}\left(W_{\text {pure }} H_{2}\left(X_{y_{i}}, \mathbf{Q}\right)\right)$ are algebraic, because they come from degree-2 Hodge homology classes on a desingularization $\widetilde{X}_{y_{i}}$, which are known to be algebraic on $\widetilde{X}_{y_{i}}$.

Going back to our pseudo-effective class $\alpha \in H_{2}(X, \mathbf{R})$, we choose an imbed$\operatorname{ding} i: X \hookrightarrow \mathbf{P}^{N}$ and denote by $U$ a neighborhood of the image of $(\phi, i)$ in $Y \times \mathbf{P}^{N}$. Thus $\phi$ is the composition of the inclusion $i^{\prime}$ of $X$ into $U$ and of the first projection $p: U \rightarrow Y$. If $U$ is small enough, it retracts over $Y$ onto $X$, so that we have an isomorphism $i_{*}^{\prime}: H_{2}(X, \mathbf{R}) \stackrel{\sim}{\rightarrow} H_{2}(U, \mathbf{R})$, and for each $y \in Y$, we have an isomorphism

$$
H_{2}\left(X_{y}, \mathbf{R}\right) \stackrel{\sim}{\rightarrow} H_{2}\left(U_{y}, \mathbf{R}\right)
$$

compatible with the maps $j_{y *}$ and $j_{U_{y} *}$, where $j_{U_{y}}: U_{y} \hookrightarrow U$ is the inclusion of the fiber $U_{y}$. It thus suffices to prove that $i_{*}^{\prime} \alpha \in H_{2}(U, \mathbf{R})$ belongs to the subspace spanned by the $j_{U_{y}}\left(H_{2}\left(U_{y}, \mathbf{R}\right)\right)$, for $y \in Y$. Since $U$ is smooth, we can use currents to compute $H_{2}(U, \mathbf{R})$. In fact, $\alpha$ being written as $\lim _{k}\left[C_{k}\right]_{\text {fund }}$, where the $C_{k}$ are effective 1 -cycles on $X$ with $\mathbf{Q}$-coefficients, $i_{*}^{\prime} \alpha$ is the class of the weakly positive current $T:=\lim _{k} T_{C_{k}}$, where $T_{C_{k}}$ is the current of integration over $i^{\prime}\left(C_{k}\right)$ acting on 2-forms of $U$. The limiting current exists, upon taking a subsequence if necessary, by [De2, Chapter III, Proposition 1.23]. The current $T$ is positive, closed of type $(N+r-1, N+r-1)$, compactly supported on $U$, and it satisfies

$$
p_{*} T=0
$$


as a current of type $(r-1, r-1)$ on $Y$. Indeed, the current $p_{*} T$ is positive on $Y$ and its homology class is equal to $\phi_{*} \alpha \in H_{2}(Y, \mathbf{R})$. The vanishing (3) thus follows from Lemma 2.2. Choose a volume form $\nu$ on $Y$. We claim that locally on $U$, we have

$$
T=p^{*} \nu \wedge T^{\prime}
$$

for a current $T^{\prime}$ of type $(N, N)$ supported on $X \subset U$. This equality means that for any 2-form $\eta$ on $U$, one has $T(\eta)=T^{\prime}\left(p^{*} \nu \wedge \eta\right)$. To see this, we use the fact that $U$ is contained in the product $Y \times \mathbf{P}^{N}$. Our local holomorphic coordinates on $U$ will be of the form $\left(y_{1}, \ldots, y_{r}, z_{1}, \ldots, z_{N}\right)$ where $\left(y_{1}, \ldots, y_{r}\right)$ are local holomorphic coordinates on $Y$ and $\left(z_{1}, \ldots, z_{N}\right)$ are local holomorphic coordinates on $\mathbf{P}^{N}$. Write

$$
\begin{gathered}
T=\iota^{r+N-1} \sum_{i, j=1}^{r} f_{i j} \operatorname{int}\left(\frac{\partial}{\partial y_{i}} \wedge \frac{\partial}{\partial \bar{y}_{j}}\right)\left(d y_{1} \wedge d \bar{y}_{1} \wedge \cdots \wedge d y_{r} \wedge d \bar{y}_{r}\right. \\
\left.\quad \wedge d z_{1} \wedge d \bar{z}_{1} \wedge \cdots \wedge d z_{N} \wedge d \bar{z}_{N}\right) \\
+\iota^{r+N-1} \sum_{k, l=1}^{N} g_{k l} \operatorname{int}\left(\frac{\partial}{\partial z_{k}} \wedge \frac{\partial}{\partial \bar{z}_{l}}\right)\left(d y_{1} \wedge d \bar{y}_{1} \wedge \cdots \wedge d y_{r} \wedge d \bar{y}_{r}\right. \\
\left.\quad \wedge d z_{1} \wedge d \bar{z}_{1} \wedge \cdots \wedge d z_{N} \wedge d \bar{z}_{N}\right) \\
+\iota^{r+N-1} \sum_{i=1}^{r} \sum_{k=1}^{N} h_{i k} \operatorname{int}\left(\frac{\partial}{\partial y_{i}} \wedge \frac{\partial}{\partial \bar{z}_{k}}\right)\left(d y_{1} \wedge d \bar{y}_{1} \wedge \cdots \wedge d y_{r} \wedge d \bar{y}_{r}\right. \\
\left.\wedge d z_{1} \wedge d \bar{z}_{1} \wedge \cdots \wedge d z_{N} \wedge d \bar{z}_{N}\right) \\
+\iota^{r+N-1} \sum_{i=1}^{r} \sum_{k=1}^{N} \frac{\partial}{h_{i k}} \operatorname{int}\left(\frac{\partial}{\partial \bar{y}_{i}} \wedge \frac{\partial}{\partial z_{k}}\right)\left(d y_{1} \wedge d \bar{y}_{1} \wedge \cdots \wedge d y_{r} \wedge d \bar{y}_{r}\right. \\
\left.\wedge d z_{1} \wedge d \bar{z}_{1} \wedge \cdots \wedge d z_{N} \wedge d \bar{z}_{N}\right),
\end{gathered}
$$

where $\iota^{2}=-1$ and the coefficients are distributions. The positivity condition for $T$ says that for any form $\alpha=\sum_{i=1}^{r} \alpha_{i} d y_{i}+\sum_{k=1}^{N} \beta_{k} d z_{k}$ of type $(1,0)$, one has $T(\iota \alpha \wedge \bar{\alpha}) \geq 0$, or equivalently that the Hermitian block matrix

$$
M=\left(\begin{array}{ll}
\left(f_{i j}\right) & \left(h_{i k}\right) \\
\left(\overline{h_{k i}}\right) & \left(g_{k l}\right)
\end{array}\right)
$$

is a semi-positive Hermitian matrix of distributions, meaning that for any $(r+$ $N)$-uple $Z=\left(Z_{r}, Z_{N}\right)$ of complex functions, with $Z_{r}=\left(\alpha_{i}\right)_{1 \leq i \leq r}$ and $Z_{N}=$ $\left(\beta_{k}\right)_{1 \leq k \leq N},{ }^{t} \bar{Z} M Z$ is a measure on $U$. 
Since $p_{*} T=0$, we get that for any form $\alpha=\sum_{i=1}^{r} \alpha_{i} d y_{i}$ on $Y$, one has $T\left(p^{*}(\iota \alpha \wedge \bar{\alpha})\right)=0$. As this is the integral over $U$ of the measure ${ }^{t} Z_{r}\left(f_{i j}\right) Z_{r}$, where $Z_{r}=\left(\alpha_{i}\right)$, we conclude that this measure is 0 , that is $f_{i j}=0$ for all $i, j \in\{1, \ldots, r\}$. The semi-positivity of $M$ implies then $\left(h_{i k}\right)=0$, so that

$$
\begin{aligned}
T=\iota^{r+N-1} \sum_{k, l=1}^{N} g_{k l} \operatorname{int}\left(\frac{\partial}{\partial z_{k}} \wedge \frac{\partial}{\partial \bar{z}_{l}}\right)\left(d y_{1} \wedge d \bar{y}_{1}\right. & \wedge \\
& \cdots \wedge d y_{r} \wedge d \bar{y}_{r} \\
& \left.\wedge d z_{1} \wedge d \bar{z}_{1} \wedge \cdots \wedge d z_{N} \wedge d \bar{z}_{N}\right) .
\end{aligned}
$$

Assuming that $\nu=\iota^{r} d y_{1} \wedge d \bar{y}_{1} \wedge \cdots \wedge d y_{r} \wedge d \bar{y}_{r}$, this gives the desired result with

$$
T^{\prime}=\iota^{N-1} \sum_{k, l=1}^{N} g_{k l} \operatorname{int}\left(\frac{\partial}{\partial z_{k}} \wedge \frac{\partial}{\partial \bar{z}_{l}}\right)\left(d z_{1} \wedge d \bar{z}_{1} \wedge \cdots \wedge d z_{N} \wedge d \bar{z}_{N}\right) .
$$

Lemma 4.4. The subspace $K:=\bigcap_{y \in Y} \operatorname{Ker}\left(H^{2}(U, \mathbf{R}) \rightarrow H^{2}\left(U_{y}, \mathbf{R}\right)\right)$ is the set of de Rham cohomology classes of closed 2 -forms on $U$ vanishing on all fibers $U_{y}$.

Proof. Let $\alpha \in K$. Then for any $y \in Y$, there is an open subset $V_{y} \subset Y$ containing $y$ such that $\left.\alpha\right|_{U_{V y}}=0$ (we use for this the fact that for any $y \in Y$, there is a neighborhood $W_{y}$ of $y$ in $Y$ such that $U_{W_{y}}$ has the same homotopy type as $U_{y}$ ). The class $\alpha$ is represented by a closed 2 -form $\tilde{\alpha}$ and we have

$$
\left.\tilde{\alpha}\right|_{U_{V_{y}}}=d \beta_{y} .
$$

Let $\left(f_{i}\right)$ be a partition of unity on $Y$ relative to an open covering of $Y$ by finitely many of the $V_{y}$, say $V_{y_{1}}, \ldots, V_{y_{M}}$. Then $\tilde{\alpha}-d\left(\sum_{i}\left(p^{*} f_{i}\right) \beta_{y_{i}}\right)$ has the same class as $\tilde{\alpha}$ and it is equal to

$$
\tilde{\alpha}-\sum_{i} p^{*} f_{i} d \beta_{y_{i}}-\sum_{i} p^{*} d f_{i} \wedge \beta_{y_{i}}=-\sum_{i} p^{*} d f_{i} \wedge \beta_{y_{i}}
$$

which clearly vanishes on each fiber $U_{y}$.

We now finish the proof of Theorem 4.2. We wanted to prove that the homology class of $T$ belongs to the space spanned by the $j_{U_{y} *} H_{2}\left(U_{y}, \mathbf{R}\right)$, for $y \in Y$. The orthogonal of this space in $H^{2}(U, \mathbf{R})$ is $K$. The result is thus equivalent to saying that for any $\eta \in K$, one has $T(\eta)=0$. By Lemma 4.4, $\eta$ is the class of a closed form $\widetilde{\eta}$ vanishing on all the $U_{y}$. On the other hand, it follows from (4) that for any 2-form $\widetilde{\eta}$ on $U$ vanishing on the fibers $U_{y}$, we have $T(\widetilde{\eta})=0$. 


\section{THE DIVISOR CASE}

5.1. Weak Conjecture B for divisors. The following theorem solves Conjecture $\mathrm{B}$ in its weak form in the divisor case.

Theorem 5.1. Let $X$ and $Y$ be smooth complex projective varieties and let $\phi$ : $X \rightarrow Y$ be a morphism. Then any pseudo-effective class $\alpha \in H^{2}(X, \mathbf{R})$ such that $\phi_{*} \alpha=0$ belongs to the real vector space spanned by classes of divisors on $X$ which are contracted by $\phi$.

Proof. Using Proposition 3.1, we can assume that $\phi$ is surjective hence, $c:=$ $\operatorname{dim}(X)-\operatorname{dim}(Y) \geq 0$. If $c \geq 2$, any divisor on $X$ is contracted by $\phi$, so the theorem holds in this case. There are thus two cases to consider, namely $c=0$ and $c=1$. We treat them separately.

Case $c=0$. We have $\operatorname{dim}(X)=\operatorname{dim}(Y)=n$. Let $Z \subset X$ be the (finite) union of the divisors of $X$ contracted by $\phi$ and let $Z^{\prime} \subset Y$ be the image of $Z$ in $Y$. By construction, $\operatorname{dim}\left(Z^{\prime}\right) \leq n-2$. Set $Y^{\prime}:=Y \backslash Z$ and $X^{\prime}:=X \backslash \phi^{-1}\left(Z^{\prime}\right)$. Since $Y$ is smooth, the map

$$
\phi^{\prime}:=\left.\phi\right|_{X^{\prime}}: X^{\prime} \rightarrow Y^{\prime}
$$

is proper with finite fibers.

We now use the following Lefschetz-type result conjectured by Deligne and proved in [HL, Thm 2.1.4 and Remark 1.1.2, (iii)].

Theorem 5.2 (Hamm-Lê). Let $X_{0}$ be an $n$-dimensional smooth complex quasiprojective variety, let $\phi_{0}: X_{0} \rightarrow \mathbf{P}^{N}$ be a morphism with finite fibers, and let $L \subset \mathbf{P}^{N}$ be a linear subspace of codimension $c$, in general position. Then the pair $\left(X_{0}, \phi_{0}^{-1}(L)\right)$ is $(n-c)$-connected.

We apply this statement to the composition of $\phi^{\prime}: X^{\prime} \rightarrow Y^{\prime}$ and an embedding of $Y$ into some $\mathbf{P}^{N}$. We conclude that for a general complete intersection surface $S_{Y} \subset Y$, with inverse image $S_{X} \subset X$, the pair $\left(X^{\prime}, S_{X}^{\prime}\right)$ is 2-connected, where $S_{X}^{\prime}:=X^{\prime} \cap S_{X}$. It follows that the restriction map

$$
H^{2}\left(X^{\prime}, \mathbf{Q}\right) \rightarrow H^{2}\left(S_{X}^{\prime}, \mathbf{Q}\right)
$$

is injective. 
Note that by Bertini's theorem, both surfaces $S_{X}$ and $S_{Y}$ are smooth. Denoting by $\phi_{S}: S_{X} \rightarrow S_{Y}$ the restriction of $\phi$ to $S_{X}$, we have, for any class $\alpha \in H^{2}(X, \mathbf{R})$ such that $\phi_{*} \alpha=0$ in $H^{2}(Y, \mathbf{R})$,

$$
\left(\phi_{S}\right)_{*}\left(\left.\alpha\right|_{S_{X}}\right)=0 \text { in } H^{2}\left(S_{Y}, \mathbf{R}\right) .
$$

Indeed, using the smoothness of $X, Y, S_{X}$, and $S_{Y}$, we easily show that

$$
\left.\left(\phi_{*} \alpha\right)\right|_{S_{Y}}=\left(\phi_{S}\right)_{*}\left(\left.\alpha\right|_{S_{X}}\right) \quad \text { in } H^{2}\left(S_{Y}, \mathbf{R}\right),
$$

so (5) follows from the vanishing of $\phi_{*} \alpha$.

Assume furthermore that $\alpha$ is a pseudo-effective class on $X$. Then $\left.\alpha\right|_{S_{X}}$ is a pseudo-effective class on $S_{X}$ since $S_{X}$ is moving in $X$, and we just proved that it is annihilated by $\phi_{S *}$. By Theorem 4.1, we conclude that the class $\left.\alpha\right|_{S_{X}}$ belongs to the real vector space spanned by classes of curves in $S_{X}$ contracted by $\phi$, hence that the class $\left.\alpha\right|_{S_{X}^{\prime}}$ is equal to 0 , since $\phi_{S}$ is finite on $S_{X}^{\prime}$. We thus proved that the class $\left.\alpha\right|_{X^{\prime}} \in H^{2}\left(X^{\prime}, \mathbf{R}\right)$ vanishes in $H^{2}\left(S_{X}^{\prime}, \mathbf{R}\right)$.

Since the restriction map $H^{2}\left(X^{\prime}, \mathbf{R}\right) \rightarrow H^{2}\left(S_{X}^{\prime}, \mathbf{R}\right)$ is injective, we conclude $\left.\alpha\right|_{X^{\prime}}=0$. We finally use the following easy fact ([V1, 11.1.2]).

Lemma 5.3. The kernel of the restriction map $H^{2}(X, \mathbf{R}) \rightarrow H^{2}\left(X^{\prime}, \mathbf{R}\right)$ is $s$ panned by classes of divisors contained in $X \backslash X^{\prime}$.

By construction, these divisors are also the divisors contracted by $\phi$ hence, this proves the theorem in the case $c=0$.

Case $c=1$. We now have $\operatorname{dim}(Y)=\operatorname{dim}(X)-1$ and the general fibers $X_{y}:=$ $\phi^{-1}(y)$ of $\phi: X \rightarrow Y$ are smooth curves. We first observe the following.

Lemma 5.4. Let $\psi: \widetilde{X} \rightarrow Z$ be a smooth projective model of the Stein factorization of $\phi$, with induced birational morphism $\tau: \widetilde{X} \rightarrow X$. A class $\alpha \in H^{2}(X, \mathbf{R})$ is annihilated by $\phi_{*}: H^{2}(X, \mathbf{R}) \rightarrow H^{0}(Y, \mathbf{R})$ if and only if the class $\tau^{*} \alpha$ is annihilated by $\psi_{*}: H^{2}(\widetilde{X}, \mathbf{R}) \rightarrow H^{0}(Z, \mathbf{R})$.

Proof. Take a general point $y \in Y$. The restriction map $H^{0}(Y, \mathbf{R}) \rightarrow H^{0}(\{y\}, \mathbf{R})$ is an isomorphism, and the composition

$$
H^{2}(X, \mathbf{R}) \stackrel{\phi_{*}}{\rightarrow} H^{0}(Y, \mathbf{R}) \rightarrow H^{0}(\{y\}, \mathbf{R})
$$

maps $\alpha$ to the sum $d$ of the degrees $d_{i}$ of its restrictions to the components $\left(X_{y, i}\right)_{1 \leq i \leq m}$ of the smooth curve $X_{y}:=\phi^{-1}(y)$. 
Similarly, for each $i \in\{1, \ldots, m\}$, the composition

$$
H^{2}(\widetilde{X}, \mathbf{R}) \stackrel{\psi_{*}}{\rightarrow} H^{0}(Z, \mathbf{R}) \rightarrow H^{0}\left(\left\{z_{i}\right\}, \mathbf{R}\right),
$$

where $z_{i}:=\psi\left(\tau^{-1}\left(X_{y, i}\right)\right) \in Z$ is over $y$, maps $\tau^{*} \alpha$ to the degree $\widetilde{d}$ of its restriction to the smooth irreducible curve $\widetilde{X}_{z_{i}}$. We have $d=\sum_{i=1}^{m} d_{i}=m \widetilde{d}$.

It follows that $d$ vanishes if and only if $\widetilde{d}$ does. This proves the lemma.

Note also that $\tau^{*} \alpha$ belongs to the real vector space spanned by classes of divisors contracted by $\psi$ if and only if $\alpha$ belongs to the real vector space spanned by classes of divisors contracted by $\phi$. Moreover, if $\alpha$ is pseudo-effective, so is $\tau^{*} \alpha$. Combining these arguments, we are reduced to the case where $\phi: X \rightarrow Y$ has connected fibers (and $X$ and $Y$ are still smooth).

Let $Y^{\prime} \subset Y$ be the dense Zariski open subset defined by the condition that $y \in Y^{\prime}$ if and only if the fiber $X_{y}:=\phi^{-1}(y)$ is a smooth curve. Set $X^{\prime}:=$ $\phi^{-1}\left(Y^{\prime}\right) \subset X$.

Let $\alpha$ be a pseudo-effective divisor class on $X$ which is annihilated by $\phi_{*}$. Its restriction to $X^{\prime}$ belongs to the kernel of the map

$$
H^{2}\left(X^{\prime}, \mathbf{R}\right) \rightarrow H^{0}\left(Y^{\prime}, R^{2} \phi_{*}^{\prime} \mathbf{R}\right),
$$

which is the first quotient map

$$
H^{2}\left(X^{\prime}, \mathbf{R}\right) \rightarrow E_{2}^{0,2}
$$

of the Leray spectral sequence of $\phi^{\prime}$. Recalling from [D1] or [V1, II, 4.2.3] that the Leray spectral sequence of $\phi^{\prime}$ degenerates at $E_{2}$, we find that the kernel $L^{1} H^{2}\left(X^{\prime}, \mathbf{R}\right)$ of the map (6) maps onto $E_{2}^{1,1}=H^{1}\left(Y^{\prime}, R^{1} \phi_{*}^{\prime} \mathbf{R}\right)$. The kernel of this last map is $E_{2}^{2,0}=H^{2}\left(Y^{\prime}, R^{0} \phi_{*}^{\prime} \mathbf{R}\right)$.

Lemma 5.5. Let $\alpha$ be as above. Then the restricted class $\left.\alpha\right|_{X^{\prime}}$ maps to 0 in the Leray quotient $H^{1}\left(Y^{\prime}, R^{1} \phi_{*}^{\prime} \mathbf{R}\right)$.

Proof. Let $C \subset Y$ be a curve which is a complete intersection of ample hypersurfaces in general position. Set $C^{\prime}:=C \cap Y^{\prime}, X_{C}:=\phi^{-1}(C)$, and $X_{C}^{\prime}:=X_{C} \cap X^{\prime}$, and let $\phi_{C}^{\prime}: X_{C}^{\prime} \rightarrow C^{\prime}$ be the restriction of $\phi$. According to Theorem 5.2, the pair $\left(Y^{\prime}, C^{\prime}\right)$ is 1-connected hence, the restriction map

$$
H^{1}\left(Y^{\prime}, R^{1} \phi_{*}^{\prime} \mathbf{R}\right) \rightarrow H^{1}\left(C^{\prime}, R^{1} \phi_{C *}^{\prime} \mathbf{R}\right)
$$


is injective. It thus suffices to show that the image of $\alpha$ in $H^{1}\left(C^{\prime}, R^{1} \phi_{C *}^{\prime} \mathbf{R}\right)$ vanishes, which we do now.

The space $H^{1}\left(C^{\prime}, R^{1} \phi_{C *}^{\prime} \mathbf{R}\right)$ is the second Leray quotient of the group $H^{2}\left(X_{C}^{\prime}, \mathbf{R}\right)$. The image of $\alpha$ in this group can be obtained as well by restricting first $\alpha$ to the smooth surface $X_{C}$, and observing that the class $\alpha_{C}$ so obtained has the property that its restriction to $X_{C}^{\prime}$ belongs to the Leray level $L^{1} H^{2}\left(X_{C}^{\prime}, \mathbf{R}\right)$. On the other hand, the class $\alpha_{C}$ is pseudo-effective on $X_{C}$ and it is annihilated by the map $\phi_{C *}: H^{2}\left(X_{C}, \mathbf{R}\right) \rightarrow H^{0}(C, \mathbf{R})$. Hence, we can apply Theorem 4.1 to $\alpha_{C}$ and conclude that it belongs to the space spanned by classes of components of fibers of $\phi_{C}: X_{C} \rightarrow C$. It follows that the restriction $\left.\alpha_{C}\right|_{X_{C}^{\prime}}$ vanishes in the Leray quotient $H^{1}\left(C^{\prime}, R^{1} \phi_{C *}^{\prime} \mathbf{R}\right)$.

Lemma 5.5 tells us that $\left.\alpha\right|_{X^{\prime}}$ belongs to the subspace

$$
E_{2}^{2,0}=H^{2}\left(Y^{\prime}, R^{0} \phi_{*}^{\prime} \mathbf{R}\right) \subset H^{2}\left(X^{\prime}, \mathbf{R}\right) .
$$

As we assumed that $\phi$ has connected fibers, $R^{0} \phi_{*}^{\prime} \mathbf{R}=\mathbf{R}$ and $H^{2}\left(Y^{\prime}, R^{0} \phi_{*}^{\prime} \mathbf{R}\right) \subset$ $H^{2}\left(X^{\prime}, \mathbf{R}\right)$ is nothing but $\phi^{\prime *} H^{2}\left(Y^{\prime}, \mathbf{R}\right) \subset H^{2}\left(X^{\prime}, \mathbf{R}\right)$.

We have the following result.

Lemma 5.6. Let $\alpha \in N^{1}(X)_{\mathbf{R}}$. Assume that its image $\beta \in H^{2}\left(X^{\prime}, \mathbf{R}\right)$ under the restriction map belongs to $\phi^{\prime *} H^{2}\left(Y^{\prime}, \mathbf{R}\right)$. Then $\beta$ belongs to the image of the composed map

$$
N^{1}(Y)_{\mathbf{R}} \stackrel{\phi^{*}}{\rightarrow} H^{2}(X, \mathbf{R}) \rightarrow H^{2}\left(X^{\prime}, \mathbf{R}\right)
$$

More precisely, we have

$$
\alpha=\phi^{*} \beta^{\prime}+\alpha^{\prime}
$$

where $\beta^{\prime} \in N^{1}(Y)_{\mathbf{R}}$ and $\alpha^{\prime}$ is an $\mathbf{R}$-linear combination of classes of divisors supported on $X \backslash X^{\prime}$.

Proof. We have $\beta=\left.\alpha\right|_{X^{\prime}}$, with $\alpha \in N S(X)_{\mathbf{R}}$ and $\beta=\phi^{\prime *} \beta^{\prime \prime}$ for some $\beta^{\prime \prime} \in$ $H^{2}\left(Y^{\prime}, \mathbf{R}\right)$. Let $l$ be an ample class on $X$, with degree $e>0$ on the general fibers of $\phi$. We have by the projection formula

$$
e \beta^{\prime \prime}=\phi_{*}^{\prime}\left(l \cup \phi^{\prime *} \beta^{\prime \prime}\right)=\phi_{*}^{\prime}(l \cup \beta)
$$

which can be rewritten as

$$
e \beta^{\prime \prime}=\left.\phi_{*}(l \cup \alpha)\right|_{Y^{\prime}} .
$$


The class $\beta^{\prime}:=\frac{1}{e} \phi_{*}(l \cup \alpha)$ belongs to $N^{1}(Y)_{\mathbf{R}}$. We thus have

$$
\beta=\phi^{\prime *} \beta^{\prime \prime}=\left.\phi^{*} \beta^{\prime}\right|_{X^{\prime}}
$$

for some $\beta^{\prime} \in N S(Y)_{\mathbf{R}}$. Recalling that $\beta=\left.\alpha\right|_{X^{\prime}}$, we get

$$
\left.\left(\phi^{*} \beta^{\prime}-\alpha\right)\right|_{X^{\prime}}=0 \text { in } H^{2}\left(X^{\prime}, \mathbf{R}\right) .
$$

By Lemma 5.3, the difference $\alpha^{\prime}:=\phi^{*} \beta^{\prime}-\alpha$ belongs to the real vector subspace of $N^{1}(X)_{\mathbf{R}}$ spanned by classes of divisors supported on $X \backslash X^{\prime}$.

Hence we proved that

$$
\alpha=\phi^{*} \beta^{\prime}-\alpha^{\prime},
$$

for some $\beta^{\prime} \in N^{1}(Y)_{\mathbf{R}}$ and some $\alpha^{\prime}$ in the real vector subspace of $N^{1}(X)_{\mathbf{R}}$ spanned by classes of divisors supported on $X \backslash X^{\prime}$.

This lemma immediately implies the theorem when $c=1$. Indeed, let $\alpha$ be a degree-2 pseudo-effective class which is annihilated by $\phi_{*}$. Using Lemmas 5.5 and 5.6, we decompose $\alpha$ as in (7). Clearly, $\phi^{*}\left(N^{1}(Y)_{\mathbf{R}}\right)$ is spanned by classes of divisors contracted by $\phi$ and similarly, the divisorial part of the complement $X \backslash X^{\prime}$, being sent by $\phi$ to the proper closed algebraic subset $Y \backslash Y^{\prime} \subset Y$, consists of subvarieties contracted by $\phi$.

5.2. Strong Conjecture B for divisors. We now show that for divisors, the strong form of Conjecture B follows from its weak form.

Theorem 5.7. Strong Conjecture B holds true for divisors $(l=1)$.

Proof. As in the proof of Theorem 5.1, we may by Proposition 3.1 assume that $\phi$ is surjective and that $c:=\operatorname{dim}(X)-\operatorname{dim}(Y)$ is 0 or 1 . We denote by $\phi: X \stackrel{\psi}{\rightarrow} Z \stackrel{h}{\rightarrow} Y$ the Stein factorization of $\phi$, where $X$ and $Y$ are smooth and $Z$ is normal.

Case $c=0$. The morphism $\phi$ is generically finite. Let $\alpha \in H^{2}(X, \mathbf{R})$ be a pseudo-effective cohomology class annihilated by $\phi_{*}: H^{2}(X, \mathbf{R}) \rightarrow H^{2}(Y, \mathbf{R})$. By Theorem 5.1, we can write $\alpha=\sum_{i} a_{i}\left[E_{i}\right]$, where the $a_{i}$ are real numbers and the $E_{i}$ are effective divisors contracted by $\phi$. In the Stein factorization, $\psi$ is birational and the $E_{i}$ are $\psi$-exceptional divisors. By a result of Lazarsfeld ([KL, Corollary 13]), the $\psi$-exceptional divisor $\sum_{i} a_{i} E_{i}$ is pseudo-effective if and only if $a_{i} \geq 0$ for all $i$. Hence $\alpha$ belongs to the convex cone spanned by classes of effective divisors of $X$ which are contracted by $\phi$. 
Case $c=1$. We use the same reduction as in the proof of [M, Theorem (1.12)]. By the flattening theorem of Hironaka or Gruson-Raynaud, we can find a desingularization $g: Z^{\prime} \rightarrow Z$ such that the induced morphism $\left(X \times{ }_{Z} Z^{\prime}\right)^{\text {main }} \rightarrow Z^{\prime}$ is flat. Let $X^{\prime}$ be the normalization of $\left(X \times{ }_{Z} Z^{\prime}\right)^{\text {main }}$. We have a commutative diagram of induced morphisms

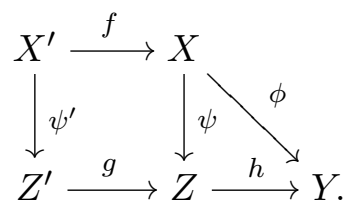

Let $\alpha \in H^{2}(X, \mathbf{R})$ be a pseudo-effective cohomology class annihilated by $\phi_{*}$ : $H^{2}(X, \mathbf{R}) \rightarrow H^{2}(Y, \mathbf{R})$. By Theorem 5.1, $\alpha$ belongs to the real vector space spanned by classes of hypersurfaces contracted by $\phi$, hence also by $\psi$. Since $X$ is smooth, $\alpha$ is in particular the class of an $\mathbf{R}$-Cartier $\mathbf{R}$-divisor on $X$, hence $f^{*} \alpha$ is the class of an $\mathbf{R}$-Cartier $\mathbf{R}$-divisor $D:=\sum_{i} a_{i} D_{i}$, where $a_{i} \in \mathbf{R}$ and the $D_{i}$ are irreducible hypersurfaces on $X^{\prime}$ contracted by $\psi^{\prime}$ (i.e., $\psi^{\prime}\left(D_{i}\right) \neq Z^{\prime}$ ).

More precisely, since $X^{\prime} \rightarrow\left(X \times{ }_{Z} Z^{\prime}\right)^{\text {main }}$ is finite and $\left(X \times_{Z} Z^{\prime}\right)^{\text {main }} \rightarrow Z^{\prime}$ is flat, the $\psi^{\prime}\left(D_{i}\right)$ are hypersurfaces in $Z^{\prime}$. For each irreducible component $E_{j}$ of $\psi^{\prime}(D)$, we write $\psi^{\prime *} E_{j}=\sum_{i} b_{i}^{j} D_{i}$. Set

$$
c_{j}:=\min _{b_{i}^{j} \neq 0} \frac{a_{i}}{b_{i}^{j}}
$$

and $E:=\sum_{j} c_{j} E_{j}$; since $Z^{\prime}$ is smooth, this an $\mathbf{R}$-Cartier $\mathbf{R}$-divisor. The $\mathbf{R}$ Cartier R-divisor $G:=D-\psi^{\prime *} E$ is then contracted by $\psi^{\prime}$. Moreover, for any irreducible component $E_{j}$ of $\psi^{\prime}(G)$, there exists an irreducible component of $\psi^{\prime-1}\left(E_{j}\right)$ not contained in $\operatorname{Supp}(G)$.

To conclude the proof of Theorem 5.7, we just need to prove the following.

Claim. The class $[E]$ in $H^{2}\left(Z^{\prime}, \mathbf{R}\right)$ is pseudo-effective.

Indeed, if the claim holds, there exists a sequence $\left(V_{n}\right)_{n}$ of effective divisors of $Z^{\prime}$ such that $\lim _{n \rightarrow \infty}\left[V_{n}\right]=[E]$ in $H^{2}\left(Z^{\prime}, \mathbf{R}\right)$. We have then

$$
\alpha=f_{*} f^{*} \alpha=\left[f_{*} D\right]=\left[f_{*} G\right]+\left[f_{*} \psi^{\prime *} E\right]=\left[f_{*} G\right]+\lim _{n \rightarrow \infty}\left[f_{*} \psi^{\prime *} V_{n}\right]
$$

hence $\alpha$ is pseudo-effective. 
Proof of the claim. Let $\varepsilon: \widetilde{X} \rightarrow X^{\prime}$ be a desingularization and set $\varphi:=\psi^{\prime} \circ \varepsilon$ : $\widetilde{X} \rightarrow Z^{\prime}$. We have $\varepsilon^{*} D=\varepsilon^{*} G+\varphi^{*} E$ and $\varepsilon^{*} G$ is an effective $\mathbf{R}$-Cartier $\mathbf{R}$-divisor contracted by $\varphi$. Moreover, for any prime divisor $P$ on $Z^{\prime}$, there exists a prime divisor $\Gamma$ on $\widetilde{X}$ such that $\varphi(\Gamma)=P$ and $\Gamma$ is not contained in $\operatorname{Supp}\left(\varepsilon^{*} G\right)$. In other words, $\varepsilon^{*} G$ is a $\varphi$-degenerate divisor in the sense of [GL, Definition 2.14].

We now consider the $\sigma$-decomposition (see for instance [GL, Definition 2.8 and Lemma 2.9])

$$
\varepsilon^{*} D=P_{\sigma}\left(\varepsilon^{*} D\right)+N_{\sigma}\left(\varepsilon^{*} D\right) .
$$

Recall that $P_{\sigma}\left(\varepsilon^{*} D\right)$ is pseudo-effective and

$$
N_{\sigma}\left(\varepsilon^{*} D\right):=\sum_{\Gamma} \sigma_{\Gamma}(D) \Gamma
$$

is an effective $\mathbf{R}$-divisor, where, fixing some ample divisor $A$ on $\widetilde{X}$, we set

$$
\sigma_{\Gamma}(D):=\min _{t>0}\left\{\operatorname{mult}_{\Gamma}\left(D^{\prime}\right) \mid D^{\prime} \sim_{\mathbf{Q}} D+t A\right\} .
$$

Let $H$ be a very ample divisor on $Z^{\prime}$. Then by definition of $N_{\sigma}$, we have

$$
N_{\sigma}\left(\varepsilon^{*} D+\varphi^{*} H\right) \leq N_{\sigma}\left(\varepsilon^{*} D\right) .
$$

On the other hand, $\varepsilon^{*} D+\varphi^{*} H=\varphi^{*}(E+H)+\varepsilon^{*} G$. We may assume that $E+H$ is effective. Then by [GL, Lemma 2.16], we have

$$
\varepsilon^{*} G \leq N_{\sigma}\left(\varepsilon^{*} D+\varphi^{*} H\right) .
$$

Thus $\varepsilon^{*} G \leq N_{\sigma}\left(\varepsilon^{*} D\right)$ hence,

$$
\varphi^{*} E=\varepsilon^{*} D-\varepsilon^{*} G=P_{\sigma}\left(\varepsilon^{*} D\right)+\left(N_{\sigma}\left(\varepsilon^{*} D\right)-\varepsilon^{*} G\right)
$$

is pseudo-effective. Thus $E$ is pseudo-effective.

\section{WEAKLY PSEUDO-EFFECTIVE CYCLES}

We turn now to Conjecture $\mathrm{C}$, a variant of Conjecture A involving weakly pseudo-effective classes. This variant is quite natural, as we already used currents in the arguments in previous sections and they seem to be the right tool to attack the problem. We will show however that it would have strong consequences for the generalized Hodge conjecture.

Let $X$ be a smooth projective variety such that $H^{i, 0}(X)=0$ for $i>0$. Equivalently, the Hodge coniveau of $H^{i}(X, \mathbf{Q})$ is at least 1 for any $i>0$. The 
following conjecture is a particular case of the generalized Hodge conjecture of Grothendieck ([Gr]).

Conjecture D (Grothendieck). If $X$ is as above, there exists a proper closed algebraic subset $Y \subset X$ such that the restriction maps $H^{i}(X, \mathbf{Q}) \rightarrow H^{i}(X \backslash Y, \mathbf{Q})$ are zero for all $i>0$.

If $n=\operatorname{dim}(X)$, by the Künneth decomposition, the conclusion is equivalent to the fact that the cycle class

$$
\left[\Delta_{X}-x \times X\right] \in H^{2 n}(X \times X, \mathbf{Q})
$$

vanishes in $H^{2 n}(X \times(X \backslash Y), \mathbf{Q})$ or, equivalently, comes from a class in $H_{2 n}(X \times$ $Y, \mathbf{Q})$.

A stronger version of Conjecture D thus asserts that there exist a proper closed algebraic subset $Y \subset X$ and an $n$-cycle $Z \in \mathscr{Z}_{n}(X \times X)_{\mathbf{Q}}$ supported on $X \times Y$ such that the class of $Z$ in $H^{2 n}(X \times X, \mathbf{Q})$ is equal to $\left[\Delta_{X}-x \times X\right]$. We prove below (Theorem 6.4) that this strong form of Conjecture D is a consequence of Conjecture $\mathrm{C}$ made in the introduction.

We begin with the following.

Proposition 6.1. Let $X$ be as above (that is, $H^{i, 0}(X)=0$ for $i>0$ ) and let $\omega \in H^{2}(X, \mathbf{Z})$ be the class of an ample line bundle on $X$. Then for $\lambda \gg 0$, the class

$$
\lambda\left(\operatorname{pr}_{1}^{*} \omega+\operatorname{pr}_{2}^{*} \omega\right)^{n-1} \cup \operatorname{pr}_{2}^{*} \omega+\left[\Delta_{X}-x \times X\right]
$$

is weakly pseudo-effective, where $\mathrm{pr}_{1}$ and $\mathrm{pr}_{2}$ are the two projections from $X \times X$ to $X$.

Proof. Let $\left(\alpha_{i}^{p, q}\right)_{i, p+q>0}$ be a basis for $H^{>0}(X, \mathbf{C})$, where $\alpha_{i}^{p, q}$ has type $(p, q)$, and let $\left(\beta_{i}^{n-p, n-q}\right)$ be the dual basis for $H^{<2 n}(X, \mathbf{C})$. By Hodge theory, these classes are represented by closed forms $\tilde{\alpha}_{i}^{p, q}$ and $\tilde{\beta}_{i}^{n-p, n-q}$ of the given type. The class $\left[\Delta_{X}-x \times X\right]$ is the cohomology class of the closed form

$$
\sum_{p, q, i} \operatorname{pr}_{1}^{*} \tilde{\beta}_{i}^{n-p, n-q} \wedge \operatorname{pr}_{2}^{*} \tilde{\alpha}_{i}^{p, q}
$$

where only couples $(p, q)$ with $p+q>0$ appear. Our assumption is $H^{i, 0}(X)=0$ for $i>0$, which by Hodge symmetry also gives $H^{0, i}(X)=0$, so only couples 
$(p, q)$ with $p>0$ and $q>0$ actually appear. The form appearing in (8) is thus a sum of forms

$$
\operatorname{Re}\left(\operatorname{pr}_{1}^{*} \tilde{\beta}^{n-p, n-q} \wedge \operatorname{pr}_{2}^{*} \tilde{\alpha}^{p, q}\right),
$$

with $p>0$ and $q>0$.

In local coordinates, every form decomposes as a sum of decomposable forms, and the fact that $p>0$ and $q>0$ implies that each term in the decomposition is of the form

$$
\tilde{\alpha}^{p, q}=d z_{i} \wedge d \bar{z}_{j} \wedge \alpha^{\prime p-1, q-1}
$$

for some indices $i$ and $j$ and some form $\alpha^{\prime p-1, q-1}$. Proposition 6.1 thus follows from the following lemma.

Lemma 6.2. Let $\omega$ be a positive $(1,1)$-form on an open subset $U$ of $\mathbf{C}^{n}$ and let $\tilde{\beta}^{n-p, n-q}$ and $\alpha^{\prime p-1, q-1}$ be, respectively, an $(n-p, n-q)$-form and $a(p-1, q-1)$ form on $U$. Set

$$
\gamma:=\operatorname{Re}\left(\operatorname{pr}_{1}^{*}\left(\tilde{\beta}^{n-p, n-q}\right) \wedge \operatorname{pr}_{2}^{*}\left(d z_{i} \wedge d \bar{z}_{j} \wedge \alpha^{\prime p-1, q-1}\right)\right) .
$$

Then there exists locally on $U \times U$ a positive real number $\lambda$ such that

$$
\lambda\left(\operatorname{pr}_{1}^{*} \omega+\operatorname{pr}_{2}^{*} \omega\right)^{n-1} \wedge \operatorname{pr}_{2}^{*} \omega \geq \gamma
$$

as real $(n, n)$-forms on $U \times U$.

Proof. For any $(n-1, n-1)$-form $\beta^{n-1, n-1}$ and any $(1,1)$-form $\beta^{1,1}$, we write

$$
\operatorname{Re}\left(\beta^{n-1, n-1} \wedge \beta^{1,1}\right)=\operatorname{Re} \beta^{n-1, n-1} \wedge \operatorname{Re} \beta^{1,1}-\operatorname{Im} \beta^{n-1, n-1} \wedge \operatorname{Im} \beta^{1,1} .
$$

Applying this to $\beta^{n-1, n-1}=\operatorname{pr}_{1}^{*}\left(\tilde{\beta}^{n-p, n-q}\right) \wedge \operatorname{pr}_{2}^{*}\left(\alpha^{\prime p-1, q-1}\right)$ and $\beta^{1,1}=\operatorname{pr}_{2}^{*}\left(d z_{i} \wedge\right.$ $\left.d \bar{z}_{j}\right)$, we obtain

$$
\begin{aligned}
\gamma= & \operatorname{Re}\left(\operatorname{pr}_{1}^{*}\left(\tilde{\beta}^{n-p, n-q}\right) \wedge \operatorname{pr}_{2}^{*}\left(\alpha^{\prime p-1, q-1}\right)\right) \wedge \operatorname{pr}_{2}^{*}\left(\operatorname{Re}\left(d z_{i} \wedge d \bar{z}_{j}\right)\right) \\
& -\operatorname{Im}\left(\operatorname{pr}_{1}^{*}\left(\tilde{\beta}^{n-p, n-q}\right) \wedge \operatorname{pr}_{2}^{*}\left(\alpha^{\prime p-1, q-1}\right)\right) \wedge \operatorname{pr}_{2}^{*}\left(\operatorname{Im}\left(d z_{i} \wedge d \bar{z}_{j}\right)\right) .
\end{aligned}
$$

The forms $\operatorname{Re}\left(\operatorname{pr}_{1}^{*}\left(\tilde{\beta}^{n-p, n-q}\right) \wedge \operatorname{pr}_{2}^{*}\left(\alpha^{\prime p-1, q-1}\right)\right)$ and $\operatorname{Im}\left(\operatorname{pr}_{1}^{*}\left(\tilde{\beta}^{n-p, n-q}\right) \wedge \operatorname{pr}_{2}^{*}\left(\alpha^{\prime p-1, q-1}\right)\right)$ are real of type $(n-1, n-1)$ on $U \times U$, hence satisfy local inequalities

$$
\begin{aligned}
& \operatorname{Re}\left(\operatorname{pr}_{1}^{*}\left(\tilde{\beta}^{n-p, n-q}\right) \wedge \operatorname{pr}_{2}^{*}\left(\alpha^{\prime p-1, q-1}\right)\right) \leq \lambda^{\prime}\left(\operatorname{pr}_{1}^{*} \omega+\operatorname{pr}_{2}^{*} \omega\right)^{n-1}, \\
& \operatorname{Im}\left(\operatorname{pr}_{1}^{*}\left(\tilde{\beta}^{n-p, n-q}\right) \wedge \operatorname{pr}_{2}^{*}\left(\alpha^{\prime p-1, q-1}\right)\right) \leq \lambda^{\prime}\left(\operatorname{pr}_{1}^{*} \omega+\operatorname{pr}_{2}^{*} \omega\right)^{n-1}
\end{aligned}
$$


for $\lambda^{\prime} \gg 0$. The forms $\operatorname{Re}\left(d z_{i} \wedge d \bar{z}_{j}\right)$ and $\operatorname{Im}\left(d z_{i} \wedge d \bar{z}_{j}\right)$ are real of type $(1,1)$ on $U$, hence satisfy local inequalities

$$
\operatorname{Re}\left(d z_{i} \wedge d \bar{z}_{j}\right) \leq \lambda^{\prime \prime} \omega \text { and } \operatorname{Im}\left(d z_{i} \wedge d \bar{z}_{j}\right) \leq \lambda^{\prime \prime} \omega
$$

for $\lambda^{\prime \prime} \gg 0$. Letting $\lambda=2 \lambda^{\prime} \lambda^{\prime \prime}$, we get from (10) the desired inequality (9).

Remark 6.3. Proposition 6.1 suggests the following question: under the same assumptions, is the class

$$
\lambda\left(\operatorname{pr}_{1}^{*} \omega+\operatorname{pr}_{2}^{*} \omega\right)^{n-1} \cup \operatorname{pr}_{2}^{*} \omega+\left[\Delta_{X}-x \times X\right]
$$

pseudo-effective for $\lambda \gg 0$ ?

As an immediate consequence of Proposition 6.1, we get the following.

Theorem 6.4. If Conjecture $C$ is true then, for any smooth projective variety $X$ such that $H^{i, 0}(X)=0$ for $i>0$, there exist a proper closed algebraic subset $Y \subset X$ and an $n$-cycle $Z \in \mathscr{Z}_{n}(X \times X)_{\mathbf{Q}}$ supported on $X \times Y$, where $n:=\operatorname{dim}(X)$, such that the class of $Z$ in $H^{2 n}(X \times X, \mathbf{Q})$ is equal to $\left[\Delta_{X}-x \times X\right]$.

Proof. Let $\omega$ be the class of an ample divisor on $X$. By Proposition 6.1, we know that for $\lambda \gg 0$, the class

$$
A:=\lambda\left(\operatorname{pr}_{1}^{*} \omega+\operatorname{pr}_{2}^{*} \omega\right)^{n-1} \wedge \operatorname{pr}_{2}^{*} \omega+\left[\Delta_{X}-x \times X\right] \in H^{2 n}(X \times X, \mathbf{Q})
$$

is weakly pseudo-effective. On the other hand, the class $A$ is clearly annihilated by $\operatorname{pr}_{2 *}: H^{2 n}(X \times X, \mathbf{Q}) \rightarrow H^{0}(X, \mathbf{Q})$. Conjecture $\mathrm{C}$ tells us that there exist $n$-dimensional subvarieties $Y_{i} \subset X \times X$ contracted by $\mathrm{pr}_{2}$ such that

$$
A=\sum_{i} \alpha_{i}\left[Y_{i}\right]
$$

for some $\alpha_{i} \in \mathbf{R}$. As $A$ is rational, this implies

$$
A=\sum_{i} \beta_{i}\left[Y_{i}\right]
$$

for some $\beta_{i} \in \mathbf{Q}$. Since the $Y_{i}$ are contracted by $\mathrm{pr}_{2}$, their images $\operatorname{pr}_{2}\left(Y_{i}\right) \subset X$ are proper closed algebraic subsets, and there exists a proper closed algebraic subset $Y \subset X$, such that $\operatorname{pr}_{2}\left(Y_{i}\right) \subset Y$ for all $i$. Enlarging $Y$ if necessary, we may furthermore assume that $Y$ is a divisor whose class is a multiple of $\omega$. Formula (11) then says that $A$ is the class of a $\mathbf{Q}$-cycle supported on $X \times Y$. On the other hand, the class $\left(\omega_{1}+\omega_{2}\right)^{n-1} \cup \omega_{2}$ is also clearly the class of a $\mathbf{Q}$-cycle supported on 
$X \times Y$. Hence we conclude that $\left[\Delta_{X}-x \times X\right]$ is the class of a $\mathbf{Q}$-cycle supported on $X \times Y$.

\section{REFERENCES}

[D1] Deligne, P., Théorème de Lefschetz et critères de dégénérescence de suites spectrales, Inst. Hautes Études Sci. Publ. Math. 35 (1968), 259-278.

[D2] Deligne, P., Théorie de Hodge II, Inst. Hautes Études Sci. Publ. Math. 40 (1971), 5-57.

[De1] Demailly, J.-P., Regularization of closed positive currents and intersection theory, $J$. Algebraic Geom. 1 (1992), 361-409.

[De2] Demailly, J.-P., Complex Analytic and Differential Geometry, available at http://wwwfourier.ujf-grenoble.fr/ demailly/manuscripts/agbook.pdf

[GL] Gongyo, Y., Lehmann, B., Reduction maps and minimal model theory, Compos. Math. 149 (2013), 295-308.

[Gr] Grothendieck, A., Hodge's general conjecture is false for trivial reasons, Topology 8 (1969), 299-303.

[HL] Hamm, H., Lê, D.-T., Lefschetz theorems on quasiprojective varieties, Bull. Soc. Math. France 113 (1985), 123-142.

[KL] Kollár, J., Larsen, M., Quotients of Calabi-Yau varieties, Algebra, arithmetic, and geometry: in honor of Yu. I. Manin. Vol. II, 179-211, Progr. Math. 270, Birkhäuser Boston, Inc., Boston, MA, 2009.

[KM] Kollár, J., Mori, S., Birational Geometry of Algebraic Varieties, with the collaboration of C. H. Clemens and A. Corti, translated from the 1998 Japanese original, Cambridge Tracts in Mathematics 134, Cambridge University Press, Cambridge, 1998.

[L] Lazarsfeld, R., Positivity in algebraic geometry I, Ergebnisse der Mathematik und ihrer Grenzgebiete 48, Springer-Verlag, Heidelberg, 2004.

[M] Mori, S., Classification of higher-dimensional varieties, in Algebraic geometry, Bowdoin, 1985 (Brunswick, Maine, 1985), 269-331, Proc. Sympos. Pure Math. 46, Part 1, Amer. Math. Soc., Providence, RI, 1987.

[V1] Voisin, C., Hodge theory and complex algebraic geometry I, II, Translated from the French original by Leila Schneps, Cambridge Studies in Advanced Mathematics, 76 and 77, Cambridge University Press, Cambridge, 2002.

[V2] Voisin, C., Coniveau 2 complete intersections and effective cones, Geom. Funct. Anal. 19 (2010), 1494-1513.

\section{Olivier Debarre}

Département de Mathématiques et Applications

\section{CNRS UMR 8553}

École Normale Supérieure

45 rue d'Ulm 
75230 Paris cedex 05, France

E-mail: olivier.debarre@ens.fr

\section{Zhi Jiang}

Département de Mathématiques

CNRS UMR 8628

Université Paris-Sud

Bâtiment 425

91405 Orsay cedex, France

E-mail: zhi.jiang@math.u-psud.fr

Claire Voisin

Centre de mathématiques Laurent Schwartz

CNRS UMR 7640

École Polytechnique

91128 Palaiseau cedex, France

E-mail: voisin@math.polytechnique.fr 